Research Paper

\title{
Proposal of predictive model on survival in unresectable pancreatic cancer receiving systemic chemotherapy
}

\author{
Noriko Ishii ${ }^{1}$, Hiroki Nishikawa ${ }^{\circledR}$, Yoshinori Iwata $^{1}$, Hirayuki Enomoto ${ }^{1}$, Hironori Tanaka ${ }^{2}$, Nobuyuki \\ Katakami ${ }^{3}$, Takashi Nishimura ${ }^{1}$, Hiroko Iijima ${ }^{1}$, Shuhei Nishiguchi ${ }^{1}$ \\ 1. Division of Hepatobiliary and Pancreatic disease, Department of Internal Medicine, Hyogo College of Medicine, Nishinomiya, Hyogo, Japan. \\ 2. Department of gastroenterology, Takarazuka municipal hospital, Takarazuka, Hyogo, Japan. \\ 3. Department of oncology, Takarazuka municipal hospital, Takarazuka, Hyogo, Japan. \\ $\square$ Corresponding author: Hiroki Nishikawa, MD, PhD, Department of Hepatobiliary and Pancreatic disease, Department of Internal Medicine, Hyogo College \\ of Medicine, Hyogo, Japan. 1-1, Mukogawacho, Nishinomiyashi, Hyogo, 663-8501, Japan. Tel: +81-798-45-6111; Fax: +81-798-45-6608, E-mail:
} nishikawa_6392_0207@hyo-med.ac.jp, Running title: Pancreatic cancer and predictive model.

(C) The author(s). This is an open access article distributed under the terms of the Creative Commons Attribution License (https://creativecommons.org/licenses/by/4.0/). See http://ivyspring.com/terms for full terms and conditions.

Received: 2019.07.30; Accepted: 2019.11.13; Published: 2020.01.01

\begin{abstract}
Aims: To construct a predictive model for overall survival $(\mathrm{OS})$ in unresectable pancreatic cancer $(\mathrm{PaC})$ undergoing systemic chemotherapy and to confirm its accuracy in an independent cohort.

Patients and methods: The training set (Ts) and the validation set (Vs) included 93 patients (median age $=71$ years) and 75 patients (median age $=76$ years). In the Ts, we examined variables linked to OS by uni- and multivariate analyses and constructed a predictive model for OS. Next, we evaluated the reproducibility of the proposed model in the Vs.

Results: In the multivariate analysis for the Ts, $P a C$ stage IV $(P=0.0020)$ and carbohydrate antigen (CA) $19-9 \geq 437.5 \mathrm{IU} / \mathrm{I}(P=0.0237)$ were identified to be significant factors associated with OS. Patients with $\mathrm{PaC}$ stage IV or not were given a score of 1 or 0 , whereas patients with CA19-9 $\geq 437.5 \mathrm{IU} / \mathrm{l}$ or $<437.5 \mathrm{IU} / \mathrm{I}$ were given a score of 1 or 0 . Sum of the point of PaC stage ( 0 or 1$)$ and CA19-9 ( 0 or 1$)$ was defined as "PaC-CA score". In the Ts, there were 16 patients with score 0,40 with score 1 and 37 with score 2, while in the Vs, there were 9 patients with score 0,32 with score 1 and 34 with score 2 . Overall $P$ values reached significance in the Ts $(P=0.0002)$, the $V_{s}(P=0.0029)$ and the combined Ts and $V s \quad(P<0.0001)$ among patients with $\mathrm{PaC}$ score 0,1 and 2 .
\end{abstract}

Conclusion: $\mathrm{PaC}-\mathrm{CA}$ score can be helpful for risk stratification in $\mathrm{PaC}$ patients undergoing systemic chemotherapy.

Key words: Unresectable pancreatic cancer, Chemotherapy, Survival, Predictive model, Validation.

\section{Introduction}

Pancreatic cancer $(\mathrm{PaC})$ is associated with a dismal prognosis, highlighted by the close relationship between cancer incidence and mortality [1-3]. The 5-year overall survival (OS) rate in $\mathrm{PaC}$ patients with advanced tumor status is extremely low [1-3]. The dismal prognosis is attributed to several causes: most importantly, numerous $\mathrm{PaC}$ patients are found in an advanced condition and the tumor is often unresectable because of its invasion to major vessels or distant metastases although several approaches to diagnosing $\mathrm{PaC}$ in its early stages have been attempted [1, 2, 4-8]. According to Japanese cancer statistical data in 2016, PaC has the seventh-highest rate of incidence and it is the fourth leading cause of death in Japan [9]. The Japanese clinical practice guidelines for systemic chemotherapy in unresectable $\mathrm{PaC}$ patients recommend the administration of following chemotherapeutic drugs: gemcitabine monotherapy, S-1 monotherapy, combined gemcitabine and S-1 therapy, combined nab-paclitaxel and gemcitabine therapy, or a combination chemotherapeutic regimen consisting of irinotecan, fluorouracil, oxaliplatin and leucovorin, considering baseline characteristics such as age, organ 
function, performance status (PS) and tumor status of each patient [10-15].

To predict prognosis in unresectable $\mathrm{PaC}$ patients undergoing systemic chemotherapy may be meaningful because it may be involved in clinical decision making whether to continue the treatment or not. Appropriate predictive models can offer a simple alternative in clinical sequences. We anticipate that a simple predictive model for OS in unresectable $\mathrm{PaC}$ patients will be useful in our daily clinical practice. However, to the best of our knowledge, there have been few reports regarding predictive models in unresectable $\mathrm{PaC}$ patients undergoing systemic chemotherapy, although numerous predictive models in $\mathrm{PaC}$ patients undergoing surgery has been reported [16-25]. The goal of the current study is to construct a simple predictive model for OS in unresectable $\mathrm{PaC}$ patients treated with systemic chemotherapy and to confirm its accuracy in an independent cohort.

\section{Patients and methods}

\section{Patients}

Between June 2008 and May 2018, a total of 97 patients with unresectable systemic chemotherapy naïve $\mathrm{PaC}$ patients were admitted at Hyogo College of Medicine Hospital (Division of Hepatobiliary and Pancreatic disease, Department of Internal Medicine), Hyogo, Japan. Four patients with lost to follow-up or insufficient clinical data were excluded from the analysis. Thus, a total of $93 \mathrm{PaC}$ patients were analyzed (the training set (Ts)). On the other hand, between July 2007 and January 2019, a total of 82 patients with unresectable systemic chemotherapy naïve $\mathrm{PaC}$ patients were admitted at the Division of Gastroenterology, Takarazuka municipal hospital, Hyogo, Japan. Seven patients with lost to follow-up or insufficient clinical data were also excluded from the analysis. A total of $75 \mathrm{PaC}$ patients were therefore analyzed (the validation set (Vs)).

\section{Diagnosis for $\mathrm{PaC}$}

$\mathrm{PaC}$ was diagnosed primarily according to the current guidelines [26]. In brief, abdominal ultrasonography and dynamic computed tomography (CT) of the whole pancreas was routinely performed before starting systemic chemotherapy. In cases without typical radiological tumor findings for $\mathrm{PaC}$, tumor biopsy or endoscopic ultrasonography-guided fine needle aspiration was in consideration [27]. In this study, the pathological diagnosis was confirmed in 44 cases $(47 \%)$ in the Ts and 54 cases $(66 \%)$ in the Vs.

\section{Systemic chemotherapy for PaC}

Each attending physician determined chemotherapeutic agents through discussion with colleagues. In PaC subjects without remarkable risk factors, the recommended starting dose of each chemotherapeutic drug (S-1, gemcitabine, 5 -fluorouracil or nab-paclitaxel) was administered [4, 28]. The reduced starting dose was administered to some patients based on clinical characteristics, including age, ECOG-PS, body weight, and laboratory data. During systemic chemotherapy, the dose of chemotherapeutic drugs was appropriately adjusted by each attending physician considering the severity of adverse events. In patients with adverse events, systemic chemotherapy was stopped until the clinical symptoms improved to grade 1 or 2 , and other alternative treatment regimens were in consideration. Other alternative regimens were also in consideration for subjects with poor treatment response to initial chemotherapy. Systemic chemotherapy was continued until any of the following conditions occurred: unacceptable toxicity for chemotherapy, tumor progression or the patient's wish to stop chemotherapy.

\section{Evaluation for treatment response}

Principally, evaluation for the treatment response for systemic chemotherapy was done every 2-4 months following the start of chemotherapy, based on the Response Evaluation Criteria in Solid Tumors (RECIST ver. 1.1) using radiological findings and tumor markers [29]. The most favorable treatment response was determined using the following four classifications: (i) Complete response (CR); (ii) partial response (PR); (iii) stable disease (SD); (iv) progressive disease (PD). The proportion of patients with the best treatment response rates of CR or PR was regarded as the objective tumor response rate (ORR), while that of patients with the best treatment response rates of $C R, P R$ or $S D$ was regarded as the disease control rate (DCR).

\section{Our study plan}

For the aim of our study, a Ts in whom systemic chemotherapy was performed in Hyogo college of medicine hospital $(n=93)$ was formed. In the Ts, we examined variables linked to OS by uni- and multivariate analyses and constructed a predictive model for predicting OS. Next, we evaluated the reproducibility of the proposed model in the subsequent Vs $(n=75)$, which included patients in the other hospital (Takarazuka municipal hospital). (Figure 1) Clinical data in the Ts were examined retrospectively and the constructed model was also verified in a retrospective manner for the independent Vs. Psoas muscle index (PMI) for the assessment of 
muscle mass was measured using $\mathrm{CT}$ as reported elsewhere [30].

Institutional review boards in each participating hospital approved the current study protocol (approval no. 2117 in Hyogo college of medicine hospital and 201707 in Takarazuka municipal hospital), with strict compliance for all of the provisions of the Declaration of Helsinki.

Hyogo college of medicine hospital (The training set (Ts), $n=93$ )

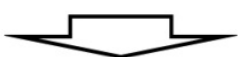

Create a predictive model

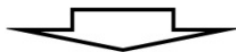

Takarazuka municipal hospital

(The validation set $(\mathrm{Vs}), \mathrm{n}=75$ )

Figure 1. Study flow chart.

\section{Statistical analysis}

A simple predictive model on survival was built from subjects in the Ts and validated in separate, independent patients in the Vs. Firstly, as mentioned above; univariate analysis was conducted to identify candidate variables among several clinical parameters to create a predictive model. The median value for each parameter was selected in order to divide the study population into two groups, which was then treated as nominal variables in the univariate analysis. Parameters with $P<0.05$ in the univariate analysis were included in the multivariate Cox hazard model. Factors with $P<0.05$ in the multivariate analysis were finally chosen as components of the novel predictive model. Based on these multivariate predictors, our proposed model was created. In the subsequent Vs, we tested the diagnostic accuracy of the created model.

OS curve was built by the Kaplan-Meier method and compared by the log-rank test. In continuous variables, the statistical comparison among groups was done by Student's $t$ test or Mann Whitney $U$ test, as applicable. Categorical variables were compared by Fisher's exact tests or Pearson $\chi^{2}$ test, as applicable. Unless otherwise stated, data were expressed as median value (range). $P$ value less than 0.05 was considered to be significant statistically with the JMP 14 (SAS Institute Inc., Cary, NC).

\section{Results}

\section{Baseline data}

The baseline data in the Ts $(n=93)$ and the Vs $(n=75)$ in this study were demonstrated in table 1 . In comparison of the Ts and the Vs, in terms of age $(P=0.0140)$, gender $(P=0.0009)$, $P S(P=0.0380)$, primary site $(P=0.0011)$, prothrombin time $(P=0.0002)$, carcinoembryonic antigen $(P=0.0117)$ and carbohydrate antigen 19-9 (CA19-9, $P=0.0170$ ), the differences in the two groups reached significance. (Table 1) The median follow-up period in the Ts and the Vs were 255 days and 217 days, respectively. In terms of initial chemotherapeutic regimens, gemcitabine monotherapy was done in 57 patients, S-1 monotherapy in 12, combined gemcitabine and S-1 therapy in 3, combined nab-paclitaxel and gemcitabine therapy in 19, uracil and tegafur therapy in 1 , and FOLFIRINOX in 1 in the Ts, while gemcitabine monotherapy was done in 44 patients, S-1 monotherapy in 8, combined gemcitabine and S-1 therapy in 6, combined nab-paclitaxel and gemcitabine therapy in 11, and FOLFIRINOX in 6 in the Vs.

Table 1. Baseline characteristics in the training set and validation set.

\begin{tabular}{|c|c|c|c|}
\hline Variables & $\begin{array}{l}\text { Training set } \\
(\mathrm{N}=93)\end{array}$ & $\begin{array}{l}\text { Validation set } \\
(\mathrm{N}=75)\end{array}$ & $P$ value \\
\hline Age (years) & $71(39-89)$ & $76(46-91)$ & 0.0140 \\
\hline Gender, male / female & $44 / 49$ & $55 / 20$ & 0.0009 \\
\hline $\begin{array}{l}\text { Performance status, } 0 / 1 \text { or } 2 / \\
\text { unknown }\end{array}$ & $75 / 18 / 0$ & $61 / 12 / 2$ & 0.0380 \\
\hline Body mass index $\left(\mathrm{kg} / \mathrm{m}^{2}\right)$ & $21(15.1-33.1)$ & $20.8(14.9-32.6)$ & 0.6385 \\
\hline $\begin{array}{l}\text { Pancreatic cancer stage, IV / } \\
\text { others }\end{array}$ & $67 / 26$ & $56 / 19$ & 0.7293 \\
\hline Maximum tumor size $(\mathrm{cm})$ & $33(9-83)$ & $35(16-110)$ & 0.2203 \\
\hline $\begin{array}{l}\text { Primary site, uncus or head / } \\
\text { body or tail }\end{array}$ & $58 / 35$ & $27 / 48$ & 0.0011 \\
\hline Total bilirubin $(\mathrm{mg} / \mathrm{dL})$ & $0.8(0.3-6.6)$ & $0.75(0.3-15.8)$ & 0.6109 \\
\hline Serum albumin $(\mathrm{g} / \mathrm{dL})$ & $3.5(1.8-4.6)$ & $3.6(2-4.8)$ & 0.1252 \\
\hline Prothrombin time (\%) & $86.2(43.5-127)$ & $78(30-116)$ & 0.0002 \\
\hline Platelet count $\left(\times 10^{4} / \mathrm{mm}^{3}\right)$ & $20.8(7.1-61)$ & $19.5(8-49.3)$ & 0.1350 \\
\hline White blood cell $\left(\times 10^{3} / \mu \mathrm{l}\right)$ & $6.11(2.54-29.76)$ & $6.36(2.43-36.24)$ & 0.1274 \\
\hline Hemoglobin (g/dl) & $11.7(7.5-15.7)$ & $11.9(1.4-17.3)$ & 0.7897 \\
\hline Serum creatinine $(\mathrm{mg} / \mathrm{dl})$ & $0.65(0.28-7.41)$ & $0.77(0.43-1.53)$ & 0.4275 \\
\hline $\mathrm{C}$ reactive protein $(\mathrm{mg} / \mathrm{dl})$ & $0.6(0-22.0)$ & $0.8(0-22.51)$ & 0.0888 \\
\hline AST (IU/L) & $26(11-265)$ & $22(2-149)$ & 0.7497 \\
\hline ALT (IU/L) & $29(6-289)$ & $23(6-294)$ & 0.5417 \\
\hline $\operatorname{ALP}(\mathrm{IU} / \mathrm{L})$ & 361 (119-1982) & $365(131-2104)$ & 0.5326 \\
\hline GGT (IU/L) & $100(11-1023)$ & $89(14-1315)$ & 0.2586 \\
\hline Amylase (IU/L) & $63.5(7-357)$ & $66.5(23-583)$ & 0.3758 \\
\hline CEA (IU/L) & $4.25(1.2-286.1)$ & $7.7(0.8-710)$ & 0.0117 \\
\hline CA19-9 (IU/L) & $437.5(0.6-42414)$ & $1344(0.2-500000)$ & 0.0170 \\
\hline $\begin{array}{l}\text { Psoas muscle index }\left(\mathrm{cm}^{2} / \mathrm{m}^{2} \text {, }\right. \\
\text { male) }\end{array}$ & $\begin{array}{l}2.576 \\
(1.340-5.708)\end{array}$ & $\begin{array}{l}2.125 \\
(0.703-8.011)\end{array}$ & 0.0788 \\
\hline $\begin{array}{l}\text { Psoas muscle index }\left(\mathrm{cm}^{2} / \mathrm{m}^{2} \text {, }\right. \\
\text { female) }\end{array}$ & $\begin{array}{l}1.947 \\
(0.509-4.662)\end{array}$ & $\begin{array}{l}1.890 \\
(0.955-3.212)\end{array}$ & 0.7126 \\
\hline
\end{tabular}


(A) Training cohort $(\mathrm{n}=93)$

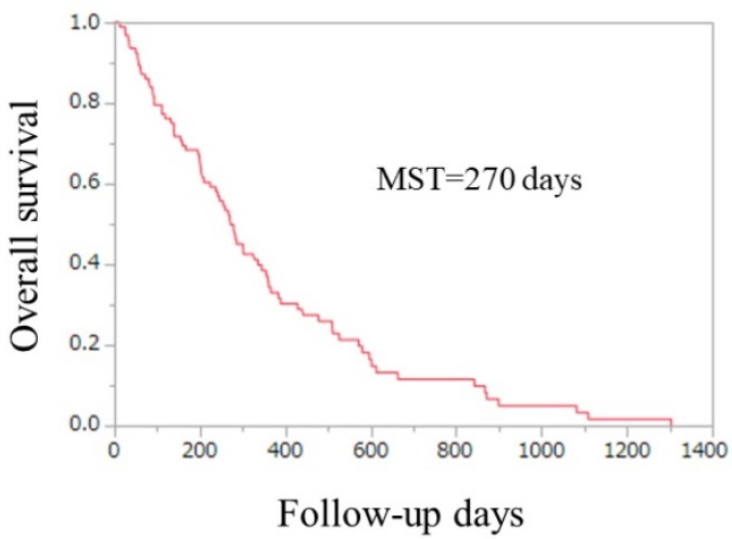

(B) Validation cohort $(\mathrm{n}=75)$

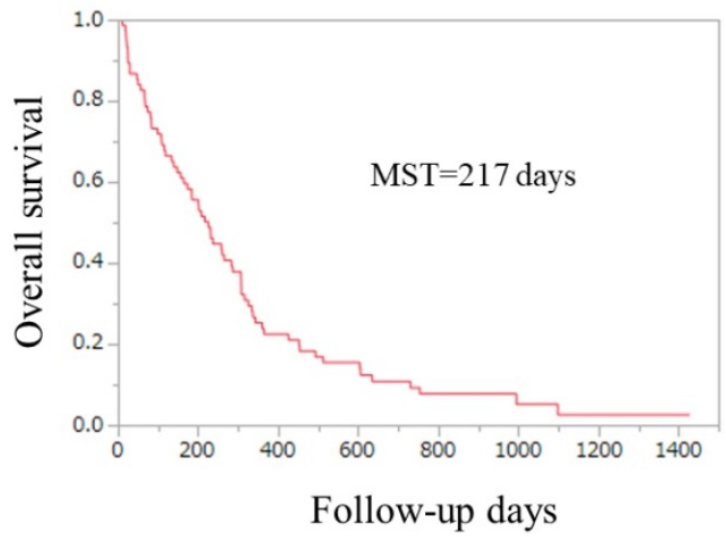

Figure 2. Overall survival in the Ts (A) and the Vs (B). MST indicates median survival time.

\section{OS in the Ts and the Vs}

Kaplan-Meier curves in the Ts and the Vs were shown in figure $2 \mathrm{~A}$ and $2 \mathrm{~B}$. The median survival time in the Ts and the Vs were 270 days and 217 days, respectively.

\section{Best tumor response during the follow-up period in the Ts and the Vs}

In the Ts, regarding the best treatment response during chemotherapy, CR was achieved in $0, P R$ in 7 , $\mathrm{SD}$ in $23, \mathrm{PD}$ in 48 and not evaluated (NE) in 15 patients. The ORR and DCR were therefore $7.5 \%$ (7/93) and $32.3 \%$ (30/93), respectively. In the Vs, regarding the best treatment response during chemotherapy, CR was achieved in $0, \mathrm{PR}$ in 4, SD in 19, PD in 40 and NE in 12 patients. The ORR and DCR were therefore $5.3 \%(4 / 75)$ and $30.7 \%$ (23/75), respectively.

\section{Causes of death}

In the Ts, $79(84.9 \%)$ patients succumbed during the observation period. All patients died because of the advanced $\mathrm{PaC}$ status. In the Vs, $70(93.3 \%)$ patients died during the observation period. All patients died because of the advanced $\mathrm{PaC}$ status.

\section{Uni- and multivariate analyses of factors associated with OS in the Ts}

Univariate analysis observed the following items as significantly associated with OS for the Ts: maximum tumor size $\geq 34 \mathrm{~mm}(P=0.0204)$; $P a C$ stage IV $(P=0.0015)$; and CĀ19-9 $\geq 437.5 \mathrm{IU} / 1 \quad(P=0.0061)$. (Table 2) The hazard ratios and $95 \%$ confidence intervals in the multivariate analysis for the three items with $P<0.05$ in the univariate analysis were presented in table 2 . PaC stage IV $(P=0.0020)$ and CA19-9 $>437.5 \mathrm{IU} / 1(P=0.0237)$ were identified to be significant prognostic factors associated with OS.
Table 2. Uni- and multivariate analyses of factors linked to overall survival in the training set.

\begin{tabular}{|c|c|c|c|c|c|}
\hline \multirow[t]{2}{*}{ Variables } & \multirow[t]{2}{*}{ Number } & \multirow{2}{*}{$\frac{\text { Univariate }}{P \text { value }}$} & \multicolumn{3}{|c|}{ Multivariate } \\
\hline & & & HR & $95 \% \mathrm{CI}$ & $P$ value \\
\hline Gender, male/female & $44 / 49$ & 0.2058 & & & \\
\hline $\begin{array}{l}\text { Psoas muscle index }\left(\mathrm{cm}^{2} / \mathrm{m}^{2}\right) \\
\text { decrease, yes/no }\end{array}$ & $43 / 45$ & 0.8079 & & & \\
\hline Age $\geq 71$ years, yes $/$ no & $48 / 45$ & 0.4562 & & & \\
\hline $\begin{array}{l}\text { Body mass index }>20.95 \\
\mathrm{~kg} / \mathrm{m}^{2} \text {, yes } / \text { no }\end{array}$ & $47 / 46$ & 0.8414 & & & \\
\hline $\begin{array}{l}\text { White blood cell count } \\
\geq 6110 \times 10^{3} / \mu \mathrm{l} \text {, yes } / \text { no }\end{array}$ & $47 / 46$ & 0.6168 & & & \\
\hline Performance status 0 , yes $/$ no & $75 / 18$ & 0.2208 & & & \\
\hline $\begin{array}{l}\text { Maximum tumor size } \geq 34 \mathrm{~mm} \text {, } \\
\text { yes } / \text { no }\end{array}$ & $46 / 47$ & 0.0204 & 1.403 & $0.885-2.232$ & 0.1489 \\
\hline Tumor stage IV, yes/no & $67 / 26$ & 0.0015 & 2.256 & $1.332-4.029$ & 0.0020 \\
\hline CA19-9 $\geq 437.5 \mathrm{IU} / 1$, yes/no & $47 / 46$ & 0.0061 & 1.715 & $1.075-2.757$ & 0.0237 \\
\hline $\begin{array}{l}\text { Serum albumin } \geq 3.6 \mathrm{~g} / \mathrm{dl} \text {, } \\
\text { yes } / \text { no }\end{array}$ & $43 / 50$ & 0.2275 & & & \\
\hline $\begin{array}{l}\text { Platelet count } \geq 20.9 \times 10^{4} / \mathrm{mm}^{3} \text {, } \\
\text { yes } / \text { no }\end{array}$ & $46 / 47$ & 0.0910 & & & \\
\hline $\begin{array}{l}\text { Prothrombin time } \geq 86.2 \% \text {, } \\
\text { yes } / \text { no }\end{array}$ & $47 / 46$ & 0.0735 & & & \\
\hline $\begin{array}{l}\mathrm{C} \text { reactive protein } \geq 0.6 \mathrm{mg} / \mathrm{dl} \text {, } \\
\text { yes } / \text { no }\end{array}$ & $46 / 47$ & 0.0750 & & & \\
\hline
\end{tabular}

\section{Our proposed predictive model}

Based on the results of multivariate analysis, patients with $\mathrm{PaC}$ stage IV were allocated a score of 1 , whereas patients with other PaC stage than stage IV were allocated a score of 0. Patients with CA19-9 $\geq 437.5 \mathrm{IU} / 1$ were allocated a score of 1 , whereas patients with CA19-9 <437.5 IU/1 were allocated a score of 0 . Sum of the point of PaC stage (0 or 1$)$ and CA19-9 (0 or 1) was defined as "PaC-CA score". $\mathrm{PaC}-\mathrm{CA}$ score therefore ranged from 0 to 2. (Table 3) We tested the predictive ability of PaC-CA score for survival in the Ts and the Vs. In the Ts, there were 16 patients with PaC-CA score 0,40 with PaC-CA score 1 and 37 with $\mathrm{PaC}-\mathrm{CA}$ score 2, while in the Vs, there were 9 patients with $\mathrm{PaC}-\mathrm{CA}$ score 0,32 with $\mathrm{PaC}-\mathrm{CA}$ score 1 and 34 with PaC-CA score 2. 


\section{(A) Training cohort}
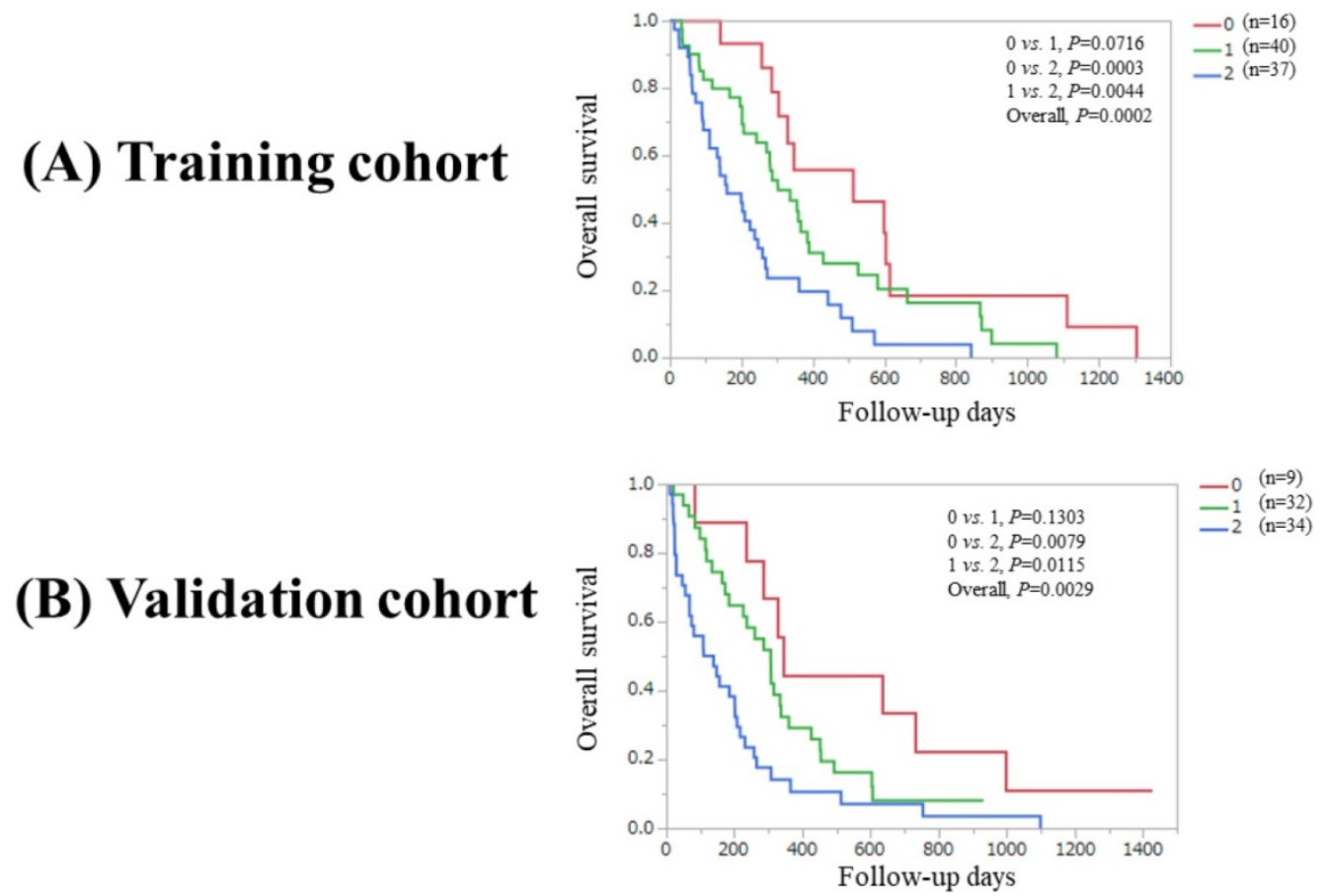

Figure 3. (A) Overall survival stratified by $\mathrm{PaC}-\mathrm{CA}$ score $(0,1$ and 2$)$ in the Ts. (B) Overall survival stratified by $\mathrm{PaC}-\mathrm{CA}$ score $(0,1$ and 2$)$ in the $\mathrm{Vs}$. PaC-CA scoring system is our newly proposed predictive model consisting of pancreatic cancer stage and CA19-9 level.

(A) Training cohort
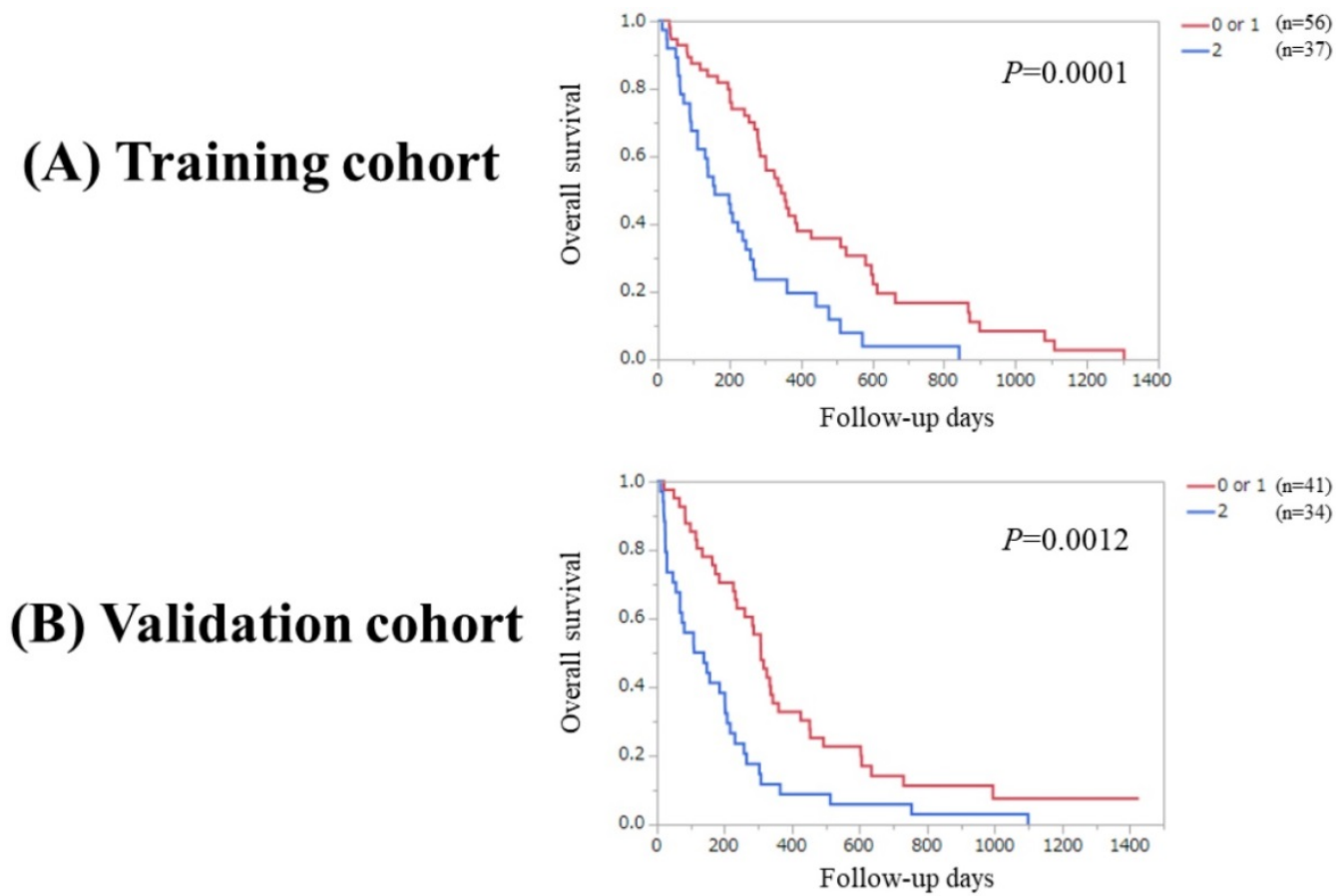

Figure 4. (A) Overall survival stratified by $\mathrm{PaC}-\mathrm{CA}$ score $(0$ or 1 and 2$)$ in the Ts. (B) Overall survival stratified by $\mathrm{PaC}-\mathrm{CA}$ score $(0$ or 1 and 2$)$ in the $\mathrm{Vs}$.

Table 3. Our proposed predictive model, called "PaC-CA score".

\begin{tabular}{llll}
\hline & Point & & \\
\hline Pancreatic cancer stage & Stage IV & Others & \\
& 1 & 0 & \\
CA19-9 & $\geq 437.5 \mathrm{IU} / \mathrm{L}$ & $<437.5 \mathrm{IU} / \mathrm{L}$ & \\
& 1 & 0 & \\
PaC-CA score & 2 & 1 & 0 \\
\hline
\end{tabular}

PaC; pancreatic cancer, $\mathrm{CA}$; carbohydrate antigen.
Overall $P$ value reached significance $(P=0.0002)$ in the Ts among patients with PaC score 0,1 and $2(P$ values between each two group: 0 vs. $1, P=0.0716 ; 0$ vs. 2, $P=0.0003$; and 1 vs. $2, P=0.0044)$. While overall $P$ value reached significance $(P=0.0029)$ in the Vs among patients with $\mathrm{PaC}$ score 0,1 and 2 ( $P$ values between each two groups: 0 vs. $1, P=0.1303 ; 0$ vs. $2, P=0.0079$; and 1 vs. 2, $P=0.0115$ ). (Figure $3 \mathrm{~A}$ and $3 \mathrm{~B}$ ). 


\section{(A) Training cohort}


Figure 5. (A) Overall survival stratified by $\mathrm{PaC}-\mathrm{CA}$ score $(0$ and 1 or 2$)$ in the Ts. (B) Overall survival stratified by PaC-CA score $(0$ and 1 or 2$)$ in the $\mathrm{Vs}$.

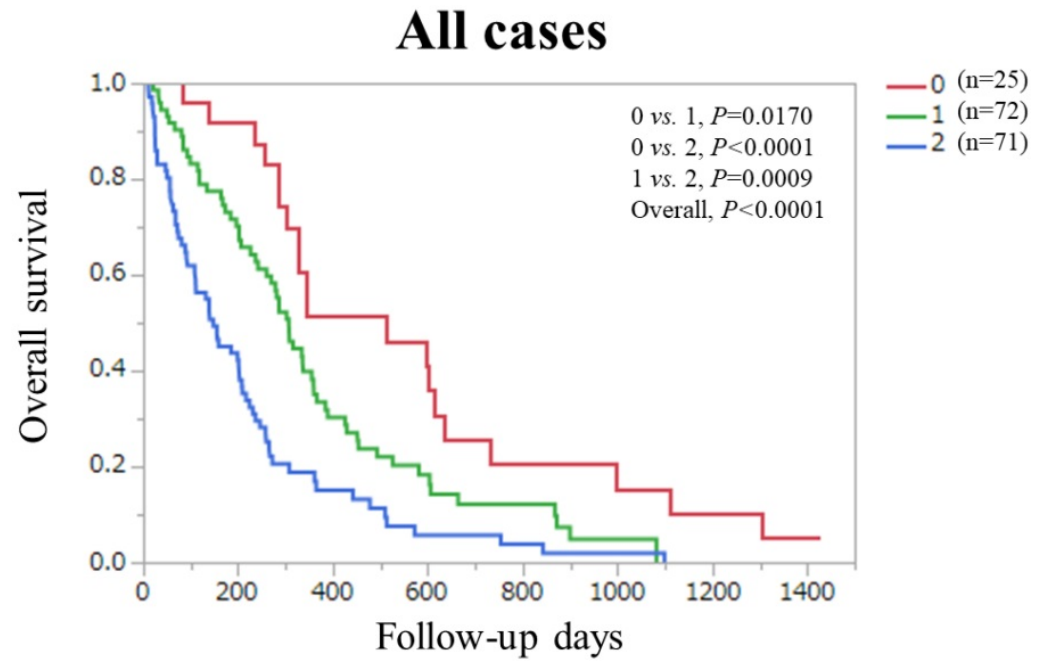

Figure 6. Overall survival stratified by $\mathrm{PaC}-\mathrm{CA}$ score $(0$ and 1 or 2$)$ in the combined $\mathrm{Ts}$ and $\mathrm{Vs}$.

Significant difference was observed between patients with $\mathrm{PaC}$ score 0 or $1(\mathrm{n}=56)$ and 2 in the Ts $(P=0.0001)$. Likewise, significant difference was observed between patients with $\mathrm{PaC}$ score 0 or 1 $(n=41)$ and 2 in the Vs $(P=0.0012)$. (Figure 4A and 4B) Significant difference was noted between patients with $\mathrm{PaC}$ score 0 and 1 or $2(\mathrm{n}=77)$ in the Ts $(P=0.0058)$. Similarly, significant difference was noted between patients with PaC score 0 and 1 or $2(n=66)$ in the Vs $(P=0.0323)$. (Figure 5A and 5B).

Finally, in the combined Ts and Vs $(n=168)$, overall $P$ value reached significance $(P<0.0001)$ among patients with $\mathrm{PaC}$ score $0(\mathrm{n}=25), 1(\mathrm{n}=72)$ and 2 $(\mathrm{n}=71)$ ( $P$ values between each two group: 0 vs. 1 ,
$P=0.0170 ; 0$ vs. $2, P<0.0001$; and 1 vs. $2, P=0.0009)$. (Figure 6).

\section{Discussion}

Decision making in the clinical settings in cancer patients may be challenging and predictive model may be helpful from the viewpoint of appropriate decision making. Here in the current study, we created a simple predictive model called "PaC-CA score", which included tumor stage and CA19-9 level. Clinicians are very familiar with these parameters in the routine clinical practice and thus PaC-CA score may be convenient and easy to access. Several predictive models such as combined 
platelet-to-lymphocyte ratio and CA 19-9 were proposed for PaC patients undergoing surgery $[16,17$, 19-23]. While, to the best of our knowledge, this is the first study for constructing a simple predictive model for $\mathrm{OS}$ in unresectable $\mathrm{PaC}$ patients undergoing systemic chemotherapy, and validating its accuracy in an independent cohort, which was a major strong point of our current analysis.

In our results, the overall significance was noted in the Ts, the Vs and the combined Ts and Vs among patients with PaC-CA score 0,1 and 2. Baseline characteristics between the Ts and the Vs were different in several parameters as shown in table 1 and our proposed model was well confirmed in the Vs and the combined Ts and Vs. These results denoted that our proposed predictive model can be helpful for risk stratification in $\mathrm{PaC}$ patients undergoing systemic chemotherapy. While between PaC-CA score 0 and 1 in the Ts and between PaC-CA score 0 and 1 in the Vs, significant difference was not noted. These results may be attributed to the small sample size of patients with PaC-CA score 0 in the Ts $(n=16)$ and the Vs $(n=9)$. In comparison of Kaplan-Meier curves between PaC-CA score 0 and 1 , that of PaC score 0 is almost persistently above that of $\mathrm{PaC}$ score 1 as presented in figure 3 , and thus we believe our predictive model is robust.

A recent study reported that CA19-9 expression in mice activated the epidermal growth factor receptor signaling, and it also cooperated with the Kras oncogene to develop aggressive pancreatic cancer [31]. Takagi, et al. demonstrated that the elevation of post-operative serum CA19-9 value was associated with an adverse outcome and reflected positivity of resection margins, and high pre-operative CA19-9 values suggested the presence of occult distant metastasis in $\mathrm{PaC}$ patients undergoing surgery [32]. Other several reports also demonstrated that CA19-9 may be involved in prognostic implication in $\mathrm{PaC}$ patients [16, 17, 33-35]. Our results were in agreement with these reports.

Recently, sarcopenia as defined by low skeletal muscle mass and low skeletal muscle function has been gaining much interest due to its prognostic impact in cancer patients [36-39]. A recent meta-analysis regarding outcome in $\mathrm{PaC}$ patients receiving surgery reported that sarcopenia was associated with increased peri-operative mortality and decreased OS [37]. While contrary to our expectations, PMI was not selected as significant factor for OS in our cohort. In our data, PMI in male ranged from 0.703 to $8.011 \mathrm{~cm}^{2} / \mathrm{m}^{2}$ (median, 2.447 $\mathrm{cm}^{2} / \mathrm{m}^{2}$ ) and PMI in female ranged from 0.509 to 4.662 $\mathrm{cm}^{2} / \mathrm{m}^{2}$ (median, $1.952 \mathrm{~cm}^{2} / \mathrm{m}^{2}$ ), which is considerably lower compared with the cutoff values of PMI for muscle mass decrease in male (6.36 $\left.\mathrm{cm}^{2} / \mathrm{m}^{2}\right)$ and female $\left(3.92 \mathrm{~cm}^{2} / \mathrm{m}^{2}\right)$ reported by Hamaguchi, et al [40]. These results may be associated with not only sarcopenia but also cancer-related cachexia [41, 42]. The fact that most of our analyzed subjects had low PMI may explain for the non-significance of PMI on OS.

Several limitations warrant mention to this study. First, our current investigation was a retrospective observational study and biases inherent to retrospective studies were unable to be completely removed, although our proposed model was verified in an independent cohort. Second, the starting chemotherapeutic drugs differed between the analyzed subjects and these differences may lead to bias for OS. Third, the sample size was relatively small for analysis (less than 100 cases both in the Ts and the Vs), potentially creating bias. Finally, our study cohort only included Japanese unresectable $\mathrm{PaC}$ patients in whom baseline characteristics such as body weight are different from $\mathrm{PaC}$ patients in Western countries [43]. Whether our current results are directly applied to different ethnic backgrounds is therefore unclear. However, the present study results demonstrated that PaC-CA score can be helpful for risk stratification in $\mathrm{PaC}$ patients treated with systemic chemotherapy.

In conclusion, our proposed predictive model can be useful in unresectable $\mathrm{PaC}$ patients undergoing systemic chemotherapy.

\section{Abbreviations}

PaC: Pancreatic cancer; OS: overall survival; CT: computed tomography; PS: performance status; RECIST: Response Evaluation Criteria in Solid Tumors; CEA: carcinoembryonic antigen; CA19-9: carbohydrate antigen 19-9; CR: complete response; PR: partial response; SD: stable disease; PD: progressive disease; ORR: objective response rate; DCR: disease control rate; NE: not evaluated; Ts: training set; Vs: validation set; PMI: psoas muscle index.

\section{Acknowledgement}

The authors would like to thank all medical staff in Hyogo college of medicine hospital and Takarazuka municipal hospital for their valuable support.

\section{Competing Interests}

The authors have declared that no competing interest exists. 


\section{References}

[1] Kamisawa T, Wood LD, Itoi T, Takaori K. Pancreatic cancer. Lancet. 2016; 388(10039): 73-85.

[2] Siegel R, Ma J, Zou Z, Jemal A. Cancer statistics, CA Cancer J Clin. 2014; 64: 9-29.

[3] Ryan DP, Hong TS, Bardeesy N. Pancreatic adenocarcinoma. N Engl J Med. 2014; 371(11): 1039-1049.

[4] Seufferlein T, Hammel P, Delpero JR, Macarulla T, Pfeiffer P, Prager GW, et al. Optimizing the management of locally advanced pancreatic cancer with a focus on induction chemotherapy: Expert opinion based on a review of current evidence. Cancer Treat Rev. 2019; 77: 1-10.

[5] Suker M, Beumer BR, Sadot E, Marthey L, Faris JE, Mellon EA, et al. FOLFIRINOX for locally advanced pancreatic cancer: a systematic review and patient-level meta-analysis. Lancet Oncol. 2016; 17(6): 801-810.

[6] Iiboshi T., Hanada K., Fukuda T., Yonehara S., Sasaki T., Chayama K. Value of cytodiagnosis using endoscopic nasopancreatic drainage for early diagnosis of pancreatic cancer: Establishing a new method for the early detection of pancreatic carcinoma in situ. Pancreas. 2012; 41: 523-529.

[7] Hanada K., Okazaki A., Hirano N., Izumi Y., Teraoka Y., Ikemoto J., et al. Diagnostic strategies for early pancreatic cancer. J. Gastroenterol. 2015; 50: 147-154.

[8] Singhi AD, Koay EJ, Chari ST, Maitra A. Early Detection of Pancreatic Cancer: Opportunities and Challenges. Gastroenterology. 2019; 156(7): 2024-2040.

[9] Kanno A, Masamune A, Hanada K, Kikuyama M, Kitano M. Advances in Early Detection of Pancreatic Cancer. Diagnostics (Basel). 2019;9(1).

[10] Yamaguchi K, Okusaka T, Shimizu K, Furuse J, Ito Y, Hanada K, et al; Committee for Revision of Clinical Guidelines for Pancreatic Cancer of the Japan Pancreas Society. Clinical Practice Guidelines for Pancreatic Cancer 2016 From the Japan Pancreas Society: A Synopsis. Pancreas. 2017; 46(5): 595-604.

[11] Kasuga A, Hamamoto Y, Takeuchi A, Kawasaki K, Suzuki T, Hirata K, et al. Positive relationship between subsequent chemotherapy and overall survival in pancreatic cancer: meta-analysis of postprogression survival for first-line chemotherapy. Cancer Chemother Pharmacol. 2017; 79(3): 595-602.

[12] Ueno H, Ioka T, Ikeda M, Ohkawa S, Yanagimoto $H$, Boku $N$, et al. Randomized phase III study of gemcitabine plus S-1, S-1 alone, or gemcitabine alone in patients with locally advanced and metastatic pancreatic cancer in Japan and Taiwan: GEST study. J Clin Oncol. 2013; 31: 1640-1648.

[13] Imaoka H, Kou T, Tanaka M, Egawa S, Mizuno N, Hijioka S, et al. Clinical outcome of elderly patients with unresectable pancreatic cancer treated with gemcitabine plus S-1, S-1 alone, or gemcitabine alone: Subgroup analysis of a randomised phase III trial, GEST study. Eur J Cancer. 2016; 54: 96-103.

[14] Kaga Y, Sunakawa Y, Kubota Y, Tagawa T, Yamamoto T, Ikusue T, et al. Early tumor shrinkage as a predictor of favorable outcomes in patients with advanced pancreatic cancer treated with FOLFIRINOX. Oncotarget. 2016; 7: 67314-67320.

[15] Von Hoff DD, Ervin T, Arena FP, Chiorean EG, Infante J, Moore M, et al. Increased survival in pancreatic cancer with nab-paclitaxel plus gemcitabine. N Engl J Med. 2013; 369: 1691-1703.

[16] Sakamoto T, Saito $H$, Amisaki M, Tokuyasu N, Honjo S, Fujiwara $Y$. Combined preoperative platelet-to-lymphocyte ratio and serum carbohydrate antigen 19-9 level as a prognostic factor in patients with resected pancreatic cancer. Hepatobiliary Pancreat Dis Int. 2019; 18(3): 278-284.

[17] Negoi I, Beuran M, Hostiuc S, El-Hussuna A, de-Madaria E. Platelet-to-lymphocyte ratio and CA19-9 are simple and informative prognostic factors in patients with resected pancreatic cancer. Hepatobiliary Pancreat Dis Int. 2019; 18(3): 203-205.

[18] Chang CF, Huang PW, Chen JS, Chen YY, Lu CH, Chang PH, et al. Prognostic Factors for Advanced Pancreatic Cancer Treated with Gemcitabine Plus S-1: Retrospective Analysis and Development of a Prognostic Model. Cancers (Basel). 2019 Jan 9;11(1). pii: E57.

[19] Balzano G, Dugnani E, Crippa S, Scavini M, Pasquale V, Aleotti F, et al. A preoperative score to predict early death after pancreatic cancer resection. Dig Liver Dis. 2017; 49(9): 1050-1056.

[20] Dasari BV, Roberts KJ, Hodson J, Stevens L, Smith AM, Hubscher SG, et al. A model to predict survival following pancreaticoduodenectomy for malignancy based on tumor site, stage and lymph node ratio. HPB (Oxford). 2016; 18(4): 332-338.

[21] Brennan MF, Kattan MW, Klimstra D, Conlon K. Prognostic nomogram for patients undergoing resection for adenocarcinoma of the pancreas. Ann Surg. 2004; 240(2): 293-298.

[22] Kanda M, Fujii T, Takami H, Suenaga M, Inokawa Y, Yamada S, et al. Combination of the serum carbohydrate antigen 19-9 and carcinoembryonic antigen is a simple and accurate predictor of mortality in pancreatic cancer patients. Surg Today. 2014; 44(9): 1692-1701.

[23] Xu J, Shi KQ, Chen BC, Huang ZP, Lu FY, Zhou MT. A nomogram based on preoperative inflammatory markers predicting the overall survival of pancreatic ductal adenocarcinoma. J Gastroenterol Hepatol. 2017; 32(7): 1394-1402.

[24] Liu L, Xu HX, He M, Wang W, Wang WQ, Wu CT, et al. A novel scoring system predicts postsurgical survival and adjuvant chemotherapeutic benefits in patients with pancreatic adenocarcinoma: Implications for AJCC-TNM staging. Surgery. 2018; 163(6): 1280-1294.

[25] Fernández A, Salgado M, García A, Buxò E, Vera R, Adeva J, et al. Prognostic factors for survival with nab-paclitaxel plus gemcitabine in metastatic pancreatic cancer in real-life practice: the ANICE-PaC study. BMC Cancer. 2018 Nov 29; 18(1): 1185

[26] Yamaguchi K, Okusaka T, Shimizu K, Furuse J, Ito Y, Hanada K, et al. Committee for revision of clinical guidelines for pancreatic cancer of Japan Pancreas Society EBM-based clinical guidelines for pancreatic cancer (2013) issued by the japan pancreas society: A synopsis. Jpn J Clin Oncol. 2014; 44: 883-888.

[27] Hewitt MJ, McPhail MJ, Possamai L, Dhar A, Vlavianos P, Monahan KJ. EUS-guided FNA for diagnosis of solid pancreatic neoplasms: A meta-analysis. Gastrointest Endosc. 2012; 75: 319-331.

[28] Springfeld C, Jäger D, Büchler MW, Strobel O, Hackert T, Palmer DH, et al. Chemotherapy for pancreatic cancer. Presse Med. 2019 Mar; 48(3 Pt 2): e159-e174.

[29] Eisenhauer EA, Therasse P, Bogaerts J, Schwartz LH, Sargent D, Ford R, et al. New response evaluation criteria in solid tumours: Revised RECIST guideline. (version 1.1) Eur J Cancer. 2009; 45: 228-247.

[30] Ishii N, Iwata $Y$, Nishikawa $H$, Enomoto H, Aizawa N, Ishii A, et al. Effect of pretreatment psoas muscle mass on survival for patients with unresectable pancreatic cancer undergoing systemic chemotherapy. Oncol Lett. 2017; 14(5): 6059-6065.

[31] Engle DD, Tiriac H, Rivera KD, Pommier A, Whalen S, Oni TE, et al. The glycan CA19-9 promotes pancreatitis and pancreatic cancer in mice. Science. 2019 Jun 21; 364(6446): 1156-1162.

[32] Takagi C, Kikuchi Y, Shirakawa H, Hoshimoto S, Tomikawa M, Ozawa I, et al. Predictive Factors for Elevated Postoperative Carbohydrate Antigen 19-9 Levels in Patients With Resected Pancreatic Cancer. Anticancer Res. 2019; 39(6): 3177-3183

[33] Gu X, Zhou R, Li C, Liu R, Zhao Z, Gao Y, et al. Preoperative maximum standardized uptake value and carbohydrate antigen 19-9 were independent predictors of pathological stages and overall survival in Chinese patients with pancreatic duct adenocarcinoma. BMC Cancer. 2019; 19(1): 456.

[34] Truty MJ, Kendrick ML, Nagorney DM, Smoot RL, Cleary SP, Graham RP, et al. Factors Predicting Response, Perioperative Outcomes, and Survival Following Total Neoadjuvant Therapy for Borderline/Locally Advanced Pancreatic Cancer. Ann Surg. 2019 Apr 5. doi: 10.1097/SLA.0000000000003284. [Epub ahead of print]

[35] Aoki S, Motoi F, Murakami Y, Sho M, Satoi S, Honda G, et al; Multicenter Study Group of Pancreatobiliary Surgery (MSG-PBS). Decreased serum carbohydrate antigen 19-9 levels after neoadjuvant therapy predict a better prognosis for patients with pancreatic adenocarcinoma: a multicenter case-control study of 240 patients. BMC Cancer. 2019; 19(1): 252.

[36] Aleixo GFP, Williams GR, Nyrop KA, Muss HB, Shachar SS. Muscle composition and outcomes in patients with breast cancer: meta-analysis and systematic review. Breast Cancer Res Treat. 2019 Jul 11. doi: 10.1007/s10549-019-05352-3. [Epub ahead of print]

[37] Bundred J, Kamarajah SK, Roberts KJ. Body composition assessment and sarcopenia in patients with pancreatic cancer: a systematic review and meta-analysis. HPB (Oxford). 2019 Jun 29. pii: S1365-182X(19)30568-4. doi: 10.1016/j.hpb.2019.05.018. [Epub ahead of print]

[38] Nishimura JM, Ansari AZ, D'Souza DM, Moffatt-Bruce SD, Merritt RE, Kneuertz PJ. Computed Tomography-Assessed Skeletal Muscle Mass as a Predictor of Outcomes in Lung Cancer Surgery. Ann Thorac Surg. 2019 Jun 19. pii: S0003-4975(19)30855-0. doi: 10.1016/j.athoracsur.2019.04.090. [Epub ahead of print]

[39] Kuwada K, Kuroda S, Kikuchi S, Yoshida R, Nishizaki M, Kagawa S, et al. Clinical Impact of Sarcopenia on Gastric Cancer. Anticancer Res. 2019; 39(5): 2241-2249.

[40] Hamaguchi Y, Kaido T, Okumura S, Kobayashi A, Hammad A, Tamai Y, et al. Proposal for new diagnostic criteria for low skeletal muscle mass based on computed tomography imaging in Asian adults. Nutrition. 2016; 32(11-12): 1200-1205.

[41] Naito T. Evaluation of the True Endpoint of Clinical Trials for Cancer Cachexia. Asia Pac J Oncol Nurs. 2019; 6(3): 227-233.

[42] Penna F, Ballarò R, Beltrà M, De Lucia S, García Castillo L, Costelli P. The Skeletal Muscle as an Active Player Against Cancer Cachexia. Front Physiol. 2019; 10: 41.

[43] Chindapasirt J. Sarcopenia in Cancer Patients. Asian Pac J Cancer Prev. 2015; 16: 8075-8077. 